\title{
Front Matter: Volume 6675
}

, "Front Matter: Volume 6675," Proc. SPIE 6675, Optical Modeling and Performance Predictions III, 667501 (25 October 2007); doi:

$10.1117 / 12.773520$

SPIE Event: Optical Engineering + Applications, 2007, San Diego, California, United SPIE. States 


\title{
PROCEEDINGS OF SPIE
}

\section{Optical Modeling and Performance Predictions III}

\author{
Mark A. Kahan \\ Editor \\ 29-30 August 2007 \\ San Diego, California, USA \\ Sponsored and Published by \\ SPIE
}

Volume 6675 
The papers included in this volume were part of the technical conference cited on the cover and title page. Papers were selected and subject to review by the editors and conference program committee. Some conference presentations may not be available for publication. The papers published in these proceedings reflect the work and thoughts of the authors and are published herein as submitted. The publisher is not responsible for the validity of the information or for any outcomes resulting from reliance thereon.

Please use the following format to cite material from this book:

Author(s), "Title of Paper," in Optical Modeling and Performance Predictions III, edited by Mark A. Kahan, Proceedings of SPIE Vol. 6675 (SPIE, Bellingham, WA, 2007) Article CID Number.

ISSN 0277-786X

ISBN 9780819468239

Published by

SPIE

P.O. Box 10, Bellingham, Washington 98227-0010 USA

Telephone +1 3606763290 (Pacific Time) · Fax +1 3606471445

SPIE.org

Copyright (C 2007, Society of Photo-Optical Instrumentation Engineers

Copying of material in this book for internal or personal use, or for the internal or personal use of specific clients, beyond the fair use provisions granted by the U.S. Copyright Law is authorized by SPIE subject to payment of copying fees. The Transactional Reporting Service base fee for this volume is $\$ 18.00$ per article (or portion thereof), which should be paid directly to the Copyright Clearance Center (CCC), 222 Rosewood Drive, Danvers, MA 01923. Payment may also be made electronically through CCC Online at copyright.com. Other copying for republication, resale, advertising or promotion, or any form of systematic or multiple reproduction of any material in this book is prohibited except with permission in writing from the publisher. The CCC fee code is $0277-786 \mathrm{X} / 07 / \$ 18.00$.

Printed in the United States of America.

Publication of record for individual papers is online in the SPIE Digital Library.

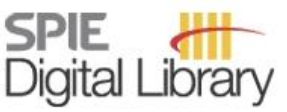

SPIEDigitallibrary.org

Paper Numbering: Proceedings of SPIE follow an e-First publication model, with papers published first online and then in print and on CD-ROM. Papers are published as they are submitted and meet publication criteria. A unique, consistent, permanent citation identifier (CID) number is assigned to each article at the time of the first publication. Utilization of CIDs allows articles to be fully citable as soon they are published online, and connects the same identifier to all online, print, and electronic versions of the publication. SPIE uses a six-digit CID article numbering system in which:

- The first four digits correspond to the SPIE volume number.

- The last two digits indicate publication order within the volume using a Base 36 numbering system employing both numerals and letters. These two-number sets start with 00, 01, 02, 03, 04, 05, $06,07,08,09,0 A, 0 B \ldots 0 Z$, followed by $10-12,20-2 Z$, etc.

The CID number appears on each page of the manuscript. The complete citation is used on the first page, and an abbreviated version on subsequent pages. Numbers in the index correspond to the last two digits of the six-digit CID number. 


\section{Contents}

vii Conference Committee
ix Introduction

\section{SESSION 1 OPTICAL MODELING ON JWST}

667503 Optical modeling activities for NASA's James Webb Space Telescope (JWST): III. Wavefront aberrations due to alignment and figure compensation [6675-02]

J. M. Howard, NASA Goddard Space Flight Ctr. (USA)

667504 Investigating the use of mutually uncorrelated acceleration spectrums to predict line of sight for JWST optical testing [6675-03]

C. L. Buttaccio, K. M. Patterson, K. A. Sweitzer, ITT Space Systems Division (USA)

\section{SESSION 2 OPTICAL MODELING ON SIM}

667505 Confusion field effects on angle tracking camera centroiding estimates for SIM PlanetQuest [6675-04]

N. Fathpour, M. Milman, C. Zhai, J. Yu, M. J. Morales, Jet Propulsion Lab. (USA)

667506 Star confusion effect on SIM PlanetQuest astrometric performance [6675-05]

C. Zhai, J. Yu, M. Milman, N. Fathpour, M. Morales, B. Nemati, M. Regehr, M. Heflin,

L. Sievers, Jet Propulsion Lab. (USA)

667507 SIM PlanetQuest interferometer real-time control system architecture [6675-06]

B. H. Kang, D. S. Bayard, G. Macala, Jet Propulsion Lab. (USA)

667508 A covariance analysis tool for assessing fundamental limits of SIM pointing performance [6675-07]

D. S. Bayard, B. H. Kang, Jet Propulsion Lab. (USA)

667509 The Instrument Model: performance prediction simulator for SIM PlanetQuest [6675-08] M. J. Morales, N. Fathpour, Jet Propulsion Lab. (USA)

SESSION 3 MODELS FOR A NEAR EARTH OBJECT OBSERVATORY AND FOR FABRICATION AND TEST OF DIAMOND TURNED ASPHERES AND/OR DOES

$66750 \mathrm{~A}$ System models used to support a space-based telescope design for discovery of near Earth objects [6675-09]

M. Lieber, J. Van Cleve, R. Arentz, H. Reitsema, R. Linfield, C. Hardesty, Ball Aerospace and Technologies Corp. (USA) 
6675 OB Stray light computations: Has nothing changed since the 1970s? [6675-11]

A. W. Greynolds, Ruda and Associates, Inc. (USA)

6675 OC Stray light design and analysis of the SNAP Telescope [6675-12]

M. J. Sholl, Univ. of California, Berkeley (USA); F. S. Grochocki, J. C. Fleming, Ball Aerospace and Technologies Corp. (USA); R. W. Besuner, P. Jelinsky, M. L. Lampton, Univ. of California, Berkeley (USA)

\section{SESSION 5 STRUCTURAL/THERMAL/OPTICAL (STOP) MODELING AND MODELS OF ELECTROSTATIC STRESS}

6675 OD Advancements in integrated structural/thermal/optical (STOP) analysis of optical systems [6675-13]

G. Stoeckel, D. Crompton, G. Perron, L-3 Communications SSG-Tinsley (USA)

6675 OE Random image motion analysis with Ivory 2.0 FE models [6675-14]

A. E. Hatheway, Alson E. Hatheway, Inc. (USA)

6675 OF On the prediction of electrostatic stresses in devices [6675-15]

G. Kloos, Meilute (Germany) and Hella KGaA Hueck and Co. (Germany)

\section{SESSION $6 \quad$ WAVE-OPTICS AND BLAZE PROFILE MODELS}

$66750 G \quad$ Algorithm for implementing an ABCD ray matrix wave-optics propagator [6675-16] J. D. Mansell, R. Praus, L. Xu, A. Seward, S. Coy, MZA Associates Corp. (USA)

$6675 \mathrm{OH} \quad$ Determining wave-optics mesh parameters for complex optical systems [6675-17] J. D. Mansell, R. Praus, S. Coy, MZA Associates Corp. (USA)

$66750 \mathrm{Ol}$ Rigorous modeling of dielectric and metallic blaze gratings in the intermediate structure regime [6675-18]

O. Sandfuchs, A. Pesch, R. Brunner, Carl Zeiss AG (Germany)

\section{SESSION 7 MODELING OF POLARIZATION BEAMSPLITTERS AND A NOVEL POLARIZATION DETECTOR}

$66750 \mathrm{~J}$ Advances in polymeric Cartesian polarizing beamsplitters and light engines employing them [6675-19]

S. Magarill, 3M Precision Optics (USA); C. L. Bruzzone, 3M Ctr. (USA)

\section{SESSION $8 \quad$ MODELS USED IN FIBER COMMUNICATIONS AND A FIBER INTERFEROMETER}

6675 OK Design of Tb/s capacity quadruply cladded optical fiber for WDM systems [6675-21]

A. Goel, Deemed Univ. (India)

6675 OL Design of wideband erbium doped fiber amplifier for WDM systems [6675-22]

A. Goel, Deemed Univ. (India); M. Goel, Gandhi Medical College (India) 
$66750 \mathrm{M}$ Processing of the signals of the single-fiber intermode interferometer with a small number of excited modes by using an electronic correlation method [6675-23]

Yu. N. Kulchin, A. D. Lantsov, Institute of Automation and Control Processes (Russia);

O. B. Vitrik, Far Eastern State Technical Univ. (Russia)

\section{POSTER SESSION}

$66750 \mathrm{~N}$ Laser line scan performance prediction [6675-24]

K. L. Mahoney, Naval Oceanographic Office (USA); O. Schofield, J. Kerfoot, Rutgers Univ. (USA); T. Giddings, J. Shirron, Metron, Inc. (USA); M. Twardowski, Wet Labs., Inc. (USA)

$667500 \quad$ Folded holographic imaging system [6675-25]

N. Ghanbari, R. K. Kostuk, M. A. Neifeld, Univ. of Arizona (USA)

6675 OP Proper: an optical propagation library for IDL [6675-27]

J. E. Krist, Jet Propulsion Lab. (USA)

$66750 Q \quad$ Finite element analysis of the LOLA receiver telescope lens [6675-28]

E. A. Matzinger, NASA Goddard Space Flight Ctr. (USA)

6675 OS Presentation, design, and application of a real-time demosaicing generic model based on a multiple channel CMOS image sampling system [6675-33]

B. Shan, T. Shen, W. Wang, Beijing Institute of Technology (China); D. T. W. Chan, Hong Kong Polytechnic Univ. (Hong Kong China)

Author Index 
Downloaded From: https://www.spiedigitallibrary.org/conference-proceedings-of-spie on 26 Apr 2023

Terms of Use: https://www.spiedigitallibrary.org/terms-of-use 


\title{
Conference Committee
}

\author{
Conference Chair
}

Mark A. Kahan, Optical Research Associates (USA)

Program Committee

James W. Bilbro, NASA Marshall Space Flight Center (USA)

Robert P. Breault, Breault Research Organization, Inc. (USA)

Gail J. Brown, Air Force Research Laboratory (USA)

Thomas G. Brown, University of Rochester (USA)

William J. Cassarly, Optical Research Associates (USA)

H. John Caulfield, Diversified Research Corporation (USA)

Helen J. Cole, NASA Marshall Space Flight Center (USA)

Keith B. Doyle, Sigmadyne, Inc. (USA)

Peter G. Eliseev, The University of New Mexico (USA)

G. Groot Gregory, Optical Research Associates (USA)

James B. Hadaway, The University of Alabama, Huntsville (USA)

Claus C. Hoff, Jet Propulsion Laboratory (USA)

George N. Lawrence, Applied Optics Research (USA)

Marie B. Levine, Jet Propulsion Laboratory (USA)

Steven P. Levitan, University of Pittsburgh (USA)

H. Angus Macleod, Thin Film Center, Inc. (USA)

Jack L. May, Northrop Grumman Corporation (USA)

Gregory J. Moore, Jet Propulsion Laboratory (USA)

James D. Moore, Jr., SRS Technologies (USA)

Steven R. Murrill, Army Research Laboratory (USA)

Sean O'Brien, Army Research Laboratory (USA)

Jefferson E. Odhner, BAE Systems (USA)

David C. Redding, Jet Propulsion Laboratory (USA)

James C. Wyant, College of Optical Sciences, University of Arizona (USA)

Richard N. Youngworth, Ball Aerospace and Technologies Corporation (USA)

Feng Zhao, Jet Propulsion Laboratory (USA)

\section{Session Chairs}

$1 \quad$ Optical Modeling on JWST

James B. Hadaway, The University of Alabama, Huntsville (USA)

2 Optical Modeling on SIM

Marie B. Levine, Jet Propulsion Laboratory (USA) 
3 Models for a Near Earth Object Observatory and for Fabrication and Test of Diamond Turned Aspheres and/or DOEs

Richard N. Youngworth, Ball Aerospace and Technologies Corporation (USA)

$4 \quad$ Modeling Stray Light

G. Groot Gregory, Optical Research Associates (USA)

5 Structural/Thermal/Optical (STOP) Modeling and Models of Electrostatic Stress

Gregory J. Moore, Jet Propulsion Laboratory (USA)

$6 \quad$ Wave-optics and Blaze Profile Models

George N. Lawrence, Applied Optics Research (USA)

7 Modeling of Polarization Beamsplitters and a Novel Polarization Detector

Thomas G. Brown, University of Rochester (USA)

8 Models Used in Fiber Communications and a Fiber Interferometer Feng Zhao, Jet Propulsion Laboratory (USA) 


\section{Introduction}

Optical systems are used just about everywhere today in systems that both image and illuminate. From eyeglasses to machine vision/robotics to automotive uses, from commercial reprographic equipment to medical instrumentation to the production of integrated circuits, and from telecommunications through Earth observations, space exploration, interferometers, nullers, and weaponry, optical systems are making a difference in our world. This conference is part of a sequence of similar conferences held in prior years that are dedicated to the optical modeling of these evolving imaging and non-imaging systems and the associated test-equipment needed to bring them forward with performance certainty. Note that models continue to be increasingly important as new missions sometimes extend beyond the ability to accurately pre-test performance.

To predict performance over such a broad range of optical systems and engineering disciplines, there are a great many mathematical methods and tools that are needed. Some need to correctly model nano-scale systems with feature sizes comparable to the wavelengths of illumination, while others may need to address precise representations of controlled LED light leakage out of purposelyroughened fibers or the fluorescent behavior of specific phosphors. Still others need to contend with components ranging from meta-materials with negative refractive index and cloaking to quantum dots, to special prisms or gratings, to large deployable telescopes where accuracies are measured in picometers or at levels approaching $1 / 10,000^{\text {th }}$ wave RMS WFE. When we add in wavelengths and configurations that range from $x$ rays to $\mathrm{THz}$, and environmental aspects spanning HEL through cryogenic in configurations from the laboratory to underwater and outer space, the number of modeling developments needed to accurately predict optical performance is immense. 
Electro-optical modeling and performance predictions also often require integrating many interdisciplinary techniques and mathematical methods with underlying physics that build upon and/or utilize (arranged by similarity, of sorts):

\author{
Geometrical and Physical Optics \\ Beam Propagation \\ Polarization \\ Radiometry \\ Stray Light/Ghosts \\ Narcissus \\ Detector Quantum Efficiency \\ Charge Diffusion \\ EMI/EMC Influences \\ Material Removal, Heat Treating \\ Testing and Calibration Models \\ MEMS and MOEMS: Electrostatics and \\ Structures \\ Mounting Stress, G-Release, Launch/ \\ Deployment \\ Vibration and Damping \\ Mechanical Influences/Scanning \\ Deformations \\ Fracture Mechanics \\ Material Factors/Lay-Up Anisotropy; \\ Inhomogeneity \\ Thermo-Elastic and Thermo-Optical Effects \\ Thermal Run-Away in IR Elements \\ System Sterilization \\ Recursive Models: T-E Changes Impact \\ Heating \\ Joint Resistance/Surface Finish and \\ Conduction Changes \\ Convective Effects and Air-Path Conditioning \\ Aero-Optics, Boundary Layers, and Shock \\ Waves \\ Integrated Models \\ Closely Coupled Thermal-Structural-Optical \\ Models \\ Radiative Damage \\ Contamination Control \\ Particulate Models \\ S/C Charging; Photopolymerization, Atomic $\mathrm{O}_{2}$ \\ Phenomenology \\ Reliability \\ Rules of Thumb \\ Diffractive, and Holographic Optics, Including \\ Coherence and Speckle \\ Fiber-Optics \\ Illumination Design, including LED/OLED \\ Models, etc. \\ Image Doubling \\ Optimization and Global Optimizers \\ Adaptive Optical Models \\ Influence Function Treatment \\ Phase/Prescription Retrieval \\ Tolerancing \\ Probabilistic Design \\ Optical Coating and Filters \\ Laser Damage Resistance \\ Ultra-Lightweight Optics/Nano-Laminates, and \\ Membrane Mirrors \\ High Impact/Shock Loading \\ Micro-Dynamics and Influences of Piece-Part \\ Inertia \\ Special Zoom/Servo Effects \\ Stress Birefringence \\ Proof Testing Models \\ Energy Absorption with Depth in Transmissive \\ Elements \\ Sources, Including THz, Fiber Lasers, and Wall- \\ Plug Efficiency Factors \\ Solar Loading \\ HEL Effects Including Survivability and \\ Hardening \\ Hole Drilling, Welding, and Laser Heat Treating \\ Aircraft/UAV Windows, Missiles, and Domes \\ Self-Induced Turbulence \\ Optical Control Systems \\ Acquisition, Pointing, and Tracking \\ Atmospheric Refraction and Scattering \\ NVR Models \\ Micro-Meteoroid Modeling, Including Models \\ of Spalling \\ Vision Systems, including HMDs and HUDs \\ Cost Models of Optical Systems \\ Scale Factors Of Use to Individual Disciplines
}

This conference brought forward new work for several of the more optical aspects in these areas. Our intent was to provide special attention to new methods of analysis that would help "anchor" various models and/or also provide parametric relationships to help correlate results with predictions. In this regard, several authors have helped to advance the state-of-the-art by contributing work that provides new insight into different aspects of optical modeling and predicting performance. 
Quite a bit of new modeling work is being done at NASA, much of it driven by JWST. John Krist (JPL) discussed PROPER, a library of IDL (Interactive Data Language) routines for simulating optical propagation in the near and far fields using Fourier-based Fresnel and angular spectrum methods. The goal of PROPER is to provide an easy to use and flexible way to study wavefront propagation, sensing, and control and it has evolved to become a free/versatile means for simulating systems that require diffraction-based rather than geometrical analyses. Limitations discussed included some real-world systems that cannot be described by simple thin lenses (e.g. some systems with multiple conic optics or those having phase aberrations that are large enough to cause aliasing in the computational grid relative to the reference sphere).

Joe Howard (GSFC) presented part three of a series describing the ongoing optical modeling activities for the James Webb Space Telescope (JWST). He investigated the aberrations resulting from alignment and figure compensation of the controllable degrees of freedom (i.e. the primary and secondary mirrors), which may be encountered during ground alignment and on-orbit commissioning of the observatory. Single field point knowledge was assumed in the corrections and aberrations over the field were reported for varying cases which helped define the general alignment problem for JWST. To prevent improper correction, knowledge of the wavefront over the field is required, where the level of necessary field knowledge depends upon the size of field and the wavefront detection noise floor. This work shows, as a rough estimation, that WF errors increase approximately linearly as a function of half field of view. This type of information is helping to guide the development of the JWST Ground Alignment and On-Orbit Commissioning Algorithms.

Chris Buttaccio, et al. (ITT) covered the expected effects of seismic and facility disturbances in testing JWST. Random power spectral density functions developed from acceleration time history data acquired at Johnson Space Center were used as FEM input disturbances to produce numerical predictions for various optical outputs, e.g., Line Of Sight (LOS), relative piece-part motions, and figure vibration. This work includes a review of the effects of uncorrelated disturbances at multiple ground interfaces of the support equipment. A particularly significant parameter noted is the contribution of LOS error due to the natural frequency of the isolation system. Design concepts without dynamic isolation, and those that have a higher bounce (axial) natural frequency that might couple into Telescope elastic modes are also addressed.

NASA's work on SIM (and TPF) has resulted in significant Modeling advancements. Nanaz Fathpour, et al. (JPL) discussed the challenges SIM faces in achieving microarcsecond accuracy for astrometry on objects as faint as 20th visual magnitude. One of the challenges seen at these dim levels is the presence of even dimmer stars inside the FOV which adds "confusion". The SIM Team parametrically looked at these effects on an Angle Tracking Camera, accounted for both shot and read noise, and computed the resultant centroid displacement 
effects on fringe shifts and reductions in visibility/performance. These studies show guide stars and grid wide angle target observations will not be corrupted by the presence of a nearby confusion field, but that confusion fields will be a problem for Dim Science targets. A parallel study shows that a possible confusion field centroiding bias of up to $\sim 100$ milliarcseconds affects performance up to a few picometers.

Chengxing Zhai, et al, (also of JPL) extended and expanded on the previous work/parametrics, providing further details on the influences of magnitude difference, spectral difference, and the angular separation between the target and confusion stars. The impact of the star confusion is found to be proportional to the intensity of the confusion star and a relatively complicated function of the separation angle, including a highly oscillating behavior and a fringe envelope suppression effect. Strategies for dealing with star confusion were also presented. For example, since the presence of additional sources in the field of view leads to inconsistent delay estimates from different channels, with sufficient signal to noise ratio, the star confusion can be detected using chi-square statistics of fringe measurements from multiple spectral channels. An interesting result of these models is that star confusion can be detected even though the interferometer cannot resolve the separation between the target and confusion stars when their spectra are sufficiently different. Noise sensitivity will eventually need to be evaluated, though combining the observations from multiple baselines is expected to be able to further reduce the astrometric error due to confusion.

Bryan Kang, et al. (JPL) covered a top-level architectural overview of SIM's realtime control system. The control system must meet challenging requirements for providing milliarcsecond class pointing and nanometer class delay-line control performance while tracking science stars as dim as 20th visual magnitude. Due to the dim science requirements and a complex control initialization processes involving target star search, acquisition, and tracking, the control system is architectured using sophisticated estimators, multiloop pathlength and angle feedforward signals, and a distributed computational infrastructure. Control objectives and requirements were presented and the necessary control sensors and actuators were discussed. The resulting overall algorithm structure is implementation using distributed processors on a ring-bus architecture.

David S. Bayard, et al. (JPL) continued the sessions on SIM with a paper that presented a performance analysis of the instrument pointing control system with emphasis on the fast steering mirror as used in combination with a multivariate control architecture to blend feedforward information with feedback information. A pointing covariance analysis tool (PCAT) was discussed which specifically analyzes systems with such complexity, and results were used to shed light on the nature of SIM performance limits and methods to potentially improve pointing performance. The results of this work do not support earlier more optimistic dimstar pointing performance predictions made based on less detailed analysis; they indicate that as the star brightness degrades from magnitude 13 to 19, the 
optimal exposure times increase from 8 to 300 seconds, and the associated pointing performance degrades from 35 to 125 mas (1-sigma radial). In simple terms, the optimal exposure times can be interpreted as trading off the contribution of centroiding error with thermo-mechanical drift error. Methods to improve performance include making the telescope aperture larger to accept more photons (reduced centroiding error), or to make the structure more stable with respect to thermal variations (less thermo-mechanical drift). The PCAT-S studies indicate that if centroiding errors are reduced, the optimal designs will favor shorter exposure times, while if thermal drift is reduced, the optimal designs will favor longer exposure times. Interestingly, reducing RWA induced jitter beyond its current 20 mas level was shown to have little effect on improving pointing performance for stars dimmer than magnitude 15, since in this range pointing errors are dominated by the centroiding and thermal drift components. An unanticipated result from the performance analysis indicates that a centroid measurement taken at a pixel crosshair gives an improvement in pointing performance worth approximately 1 magnitude in star brightness compared to a centroid taken at some arbitrary location on the pixel. This motivates designing a system, if possible, that takes all centroids in close proximity to a pixel crosshair. As stars become brighter, optimal exposure times become shorter and at some point there will be an additional benefit to smoothing centroid measurements over multiple samples. This extra smoothing is not captured in the present paper, and hence the presenter noted that the performance limits shown should not be extrapolated to bright star pointing without a separate analysis.

Mauricio Morales, et al. (JPL) concluded the sessions on SIM by discussing the SIM instrument model. This model performs single tile simulations for the SIM instrument and is used to combine the effects of errors in the different subsystems such as the science interferometer, guide interferometer, external metrology sensor, and roll sensor. The inputs to the instrument model are the sensor errors for the internal instrument measurements. These errors can be derived from testbeds or other physical models. The instrument model perturbs ideal sensors and replicates the fundamental SIM processing called delay regularization. This process reconstructs the science interferometer measurements by using additional sensor measurements. The output of the instrument model is the regularized delay, which is the principal science measurement for various observing scenarios such as wide angle grid, and narrow angle observations. Although the primary role of the instrument model has been single tile performance prediction, it also serves as variety of different system engineering activities such as validation of the SIM astrometric error budget, demonstration of SIM's capability for picometer sensing, development of instrument calibrations, analysis of system level errors, and validation of the averaging approach for the science data processing. During Instrument Integration and Testing (II\&T) it will be used to validate that the instrument meets its allocated requirements at the "super-sensor" level. Finally, during in-orbit checkout, it will be used to correlate instruments' in-orbit measurements with performance of the different components. 
Mike Lieber, et al (Ball) investigated the effects of several key system parameters related to development of a space-based observatory for discovering near-Earth objects (NEOs). This space-based mission is seen as complementary to groundbased observations for identifying objects with the potential to impact the Earth. A system model was developed from an artificial data set of 1218 NEOs with initial orbital elements generated from a probability distribution model similar to that incorporated by the NASA NEO Science Definition Team Report. By running the model over a seven-year period, the statistics of NEO detection were investigated as a function of changes to telescope parameters. This paper discusses the system model development of optical models, radiometric calculations, and some initial results from parameter studies on the engineering design, with an ultimate goal of establishing requirements for a space-based telescope, working with ground assets, that will allow a combined $>90 \%$ probability of detection.

Alan W. Greynolds (Ruda and Associates, Inc.) noted that all current commercially available codes for doing stray light computations are based on the same modified (with ray splitting and importance sampling) Monte-Carlo, non-sequential, forward, ray tracing method pioneered by the GUERAP-III program in the 1970s. The advantages and disadvantages of this method were presented first. Alan then discussed an alternative technique that is deterministic and exploits the use of bi-directional non-sequential beamlet traces. Ironically, this "new" technique is just an extension from two to three dimensions of the thesis research done by the author in the late 1970s, and the author reminds the audience/reader that with the ever increasing power of our computer systems, what was once impractical can suddenly become practical.

M. J. Sholl, et al (a combined Team from the University of California at Berkeley, and Ball) discussed stray light control in SNAP, a proposed space-based experiment designed to quantify dark energy by measuring the redshiftmagnitude diagram of supernovae and to quantify the growth of structure in the universe by measuring weak gravitational lensing over cosmological distances. The baseline SNAP telescope is an ambient temperature three-mirror anastigmat, and the goal of SNAP's stray light design is to ensure that in the 0.4 to 1.7 micron wavelength range stray radiation levels do not exceed a small fraction of Zodiacal radiation within the mission's target field near the North ecliptic pole. At visible wavelengths, the primary source of stray light was expected to be starlight scattered by the primary mirror, while in the longest wavelength NIR band thermal emission from the mirrors and structure was expected to dominate. Scattered stray light is mitigated by an internal field stop, and a cold (140K) internal aperture stop. Stray light scattered by mirror roughness and particulate contamination, as well as scattering from the telescope baffles were modeled and quantified. Allowable background levels (Zodi) were approximated in the nine photometer bands using Aldering's reddened solar spectrum and Leinert's approximations for Zodi at various sun angles. It was established that solar impingement within the baffle is unacceptable, while Earthshine is tolerable and 
Iunar illumination is inconsequential. For in-field radiation, system stray light was found to be most sensitive to contamination and roughness on the fold mirror, both using Peterson's relationships and point source transmission techniques. At $10^{\circ} \mathrm{C}$, observatory thermal emissions were found to be dominated by the SM baffle and support and mirror emission, although thermal emissions fell well within Zodiacal limits. The baseline design and analyses presented help ensure that stray light will be less than $10 \%$ of Zodiacal in all bands.

Gerhard Stoeckel, et al. (L-3 Communications SSG-Tinsley) discussed a structuralthermal-optical-program (STOP) that interfaces with both structural and optical (CODE V®) analysis codes, and achieves raytrace-generated results from the optical model. The technique is shown to provide accurate results and to help provide critical insights into system performance. Results from the Advanced Baseline Imager $A B I$ telescope were presented as an example. Al Hatheway (Alson E. Hatheway, Inc.), continued the STOP-based session by covering random image motion analysis using Ivory 2.0 FE models on a MWIR FLIR that performs both pointing and tracking system on a turret full of other sensors and systems. The payload was stabilized and vibration isolated but some vehicle disturbances get through these systems to excite the optical bench of the payload. These residual disturbances cause boresight shift and image blur of the IR image on the FLIR's cooled detector, and Ivory was used to quickly/efficiently prepare a thermal and structural dynamic NASTRAN model of the FLIR image's response to the residual disturbances. The physical prescription for the FLIR optics was put into Ivory and it calculated all the influence coefficients between the image on the detector and all the lens design variables and all the lens motions. Ivory then prepared the portion of the NASTRAN model that relates the optical element displacements to the image motions. The Ivory optical model was then attached to an elastic model of the FLIR and payload and the effects on the image of temperature changes, gravitational vector changes and residual random vibrations were then analyzed in NASTRAN.

Gerhard Kloos (Hella KGaA Hueck and Co., Germany) presented new algorithms defining how electrostatic stresses depend on the elastic and dielectric properties of materials when dielectric media are exposed to an electric field. Depending on the application, these stresses might be considered a spurious effect (e.g. confusing the measurement signal that stems from electrostriction) or an effect that should be enhanced to improve performance (e.g. in designing Maxwell-stress actuators). These stresses are complex functions of the orientation of the surface of the dielectric material with respect to the electric field and they also depend on the anisotropy of the material. The skew-symmetric part of the electrostatic Maxwell stress tensor was shown to gain importance the lower the symmetry of the dielectric material. Also, for the case of stresses at the boundary of two dielectric media, their respective electrostatic tensors and the continuity conditions that follow from Maxwell's theory of electromagnetism have to be taken into consideration. Using a phenomenological description, it was also 
shown that these stresses can be predicted, and then used for the purpose of improved instrument design.

Justin Mansell, et al. (MZA Associates Corporation), provided a pair of papers on wave-optics. The first of the pair covered an algorithm for implementing an ABCD Ray Matrix Wave-Optics Propagator, building on prior work that showed how to integrate effects that are not modeled in wave-optics with a ray matrix model. In this new work it was shown how to complete the integration of the two techniques by modifying and simplifying the Siegman $A B C D$ ray matrix decomposition. After removing the separable effects like image rotation and image inversion, the team broke the $5 \times 5$ ray matrix into two $2 \times 2$ sections (a.k.a. the $A B C D$ matrices) that correspond to two axes that are orthogonal to the propagation. They then presented a general algorithm that breaks any arbitrary $A B C D$ matrix into four simple wave-optics steps. The algorithm presented was shown to be sufficiently general so as to be able to handle image/focal planes and selected degenerate cases while being compatible with existing waveoptics codes. The method enables the rapid and accurate wave-optics modeling of the propagation of light through complex optical systems composed of simple optics. Justin (et al., MZA) then went on to discuss how to determine wave-optics mesh parameters for complex optical systems. Here, prior work covered choosing mesh parameters for a single wave-optics propagation between two effective apertures. However, most systems that require waveoptics modeling, like laser resonators with gain media, propagations through the atmosphere, and imaging systems with internal limiting apertures, have multiple apertures and phase screens that induce diffraction. This new work augments the single propagation theory to include diffraction from both apertures and phase aberrations, including systems with both large and small Fresnel number propagation, systems with a series of apertures, and systems with turbulent phase screens. These results allow a user the flexibility to correctly set up a wave-optics model of a complex optical system.

Oliver Sandfuchs, et. al. (Carl Zeiss, Germany) developed models for dielectric and metallic blaze gratings in the intermediate structure regime. Scalar and vector-based theories are often used for a first-performance estimation, however, in the intermediate structure regime, characterized by a grating period that lies in the transition region from scalar to fully electromagnetic theory, rigorous numerical simulations have been inevitable for accurate modeling of blaze structures with sawtooth-shaped profiles. A variety of electromagnetic algorithms exist to determine diffraction efficiency, such as integral equation methods, finite element methods or rigorous coupled-wave analyses, and an effect known as shadowing also occurs which has a significant influence on the diffraction efficiency of blazed gratings. The team succeeded in filling the modeling gap in the intermediate structure regime. They derived a rigorous-based semi-analytical model for dielectric gratings, and they were able to extend this model to the case of metallic reflection gratings. For both types of gratings, they found that the blaze efficiency obeys a linear dependence on the ratio of blaze wavelength to 
grating period, that these factors dominate performance in the first diffraction order, and that efficiency reductions due to finite grating structure size below the scalar limit obey an inversely linear dependence on the grating period. They also define a linear coefficient of shadowing strength and discuss its dependence on material properties. They found that electromagnetic effects dominate efficiency in the intermediate structure regime and that the differences of dielectric and metallic boundary conditions simply result in a change of the value of the linear coefficient of shadowing strength. The shadowing effect, as modeled in rayoptical theories based on deflections due to the passive blaze facet, do not appear to be the dominating effect. Efficiency reductions were smaller for metallic structures than for dielectric structures, and the reasons for these results were explained. One reason for this is that even the $2 \Pi$-phase delay generated during propagation along the passive blaze facet of a dielectric interface leads to a distortion of the wave front in the near field along the boundary. The wave front at a metallic interface, however, is less distorted and only weakly affects the blaze efficiency. Another contribution to the electromagnetic shadowing phenomenon, which is also present in both dielectric and metallic gratings, originates from the diffraction at the local blaze edge. In the ideal case of a metal grating with an infinite conductivity, the presence of the blaze edge results in the smallest but non-zero shadowing strength for the particular profile geometry investigated. These results, which provide a deeper insight into the underlying physical mechanism behind the electromagnetic effects leading to the shadowing, are expected to have significant practical importance for the optical design of hybrid systems.

Simon Magarilla, et al. (3M Precision Optics) presented new material on advances in polymeric Cartesian polarizing beamsplitters and related light engines. 3M Polarization Beam Splitter (PBS) technology has been shown to be a highly light efficient solution to the needs of LCOS projection. It also provides very high contrast and extremely uniform dark states without the use of lead in the glass prisms. The $3 \mathrm{M}$ Team discussed recent improvements in contrast performance, their increased understanding of the effects of pupil shape and size on contrast, the effects of temperature on optical performance, and improved photostability. They also suggested new unitary light-engine architectures/assemblies employing fewer parts and lower costs built upon 3M's PBSs in configurations where projection lens back focal length, high optical efficiency (throughput), high contrast, and excellent image quality were important. Associated light budgets and analyses were also presented. In addition to their use in optical imaging cores, the wide angle capabilities of these PBSs allow for extremely compact and efficient LED polarization color combiner assemblies.

Aditya Goel, (Deemed University, India) presented a pair of papers on wavelength division multiplexing (WDM) Systems. The first paper covered material on the design of Tb/s capacity quadruply cladded (QC) optical fiber for WDM systems. The enormous transmission capacity of single mode fibers cannot be fully 
utilized with conventional step index fiber, but new designs offer improvements as based upon fast/accurate numerical techniques for calculating the dispersion. Most existing numerical approaches are good enough to calculate the propagation constant, but some are not able to handle an arbitrary refractive index profile. Here, a powerful numerical technique using a quadratic finite element method is outlined, and results for the modal characteristic of a single mode optical fiber with an arbitrary refractive index profile are cross-compared with earlier step-index results. It is shown that multiple cladded fiber is better suited for wideband systems. Dispersion flattened fibers have been proposed in the past with a W-profile, but all such fiber designs have been observed to be bend sensitive in the long wavelength window, as one has to operate very close to the cutoff of the fundamental mode. Computations are shown which indicate that by properly optimizing the profile parameters, a wideband fiber can be designed where the dispersion can be kept confined with in $\pm 1.0 \mathrm{ps} / \mathrm{km}-\mathrm{nm}$ over the full wavelength span from 1280 to $1550 \mathrm{~nm}(370 \mathrm{~nm})$. The proposed QC fiber profile is shown to be capable of transmitting high speed data (>1 Tb/s), and it is expected to be relatively insensitive to bending losses, as the field, even at longer wavelengths (1550 nm), is well guided within the QC fiber structure.

Aditya, et al. (Deemed University and Gandhi Medical College, India) continued the discussion of WDM Systems by presenting a paper on the design of a wideband erbium doped fiber amplifier. The authors note that wideband optical fibers compatible with WDM transmission systems are available which can operate near the $1550 \mathrm{~nm}$ region, but their transmission capacity is limited by the slow response of conventional semiconductor amplifiers. To overcome this, researchers devised erbium doped optical amplifiers (EDFAs) which directly amplify the optical domain in a relatively narrow $(25 \mathrm{~nm})$ band centered on the $1550 \mathrm{~nm}$ region while working to devise EDFAs with broad bandwidth capabilities. The author(s) presented a technique using gain cascading of two EDFAs pumped separately at $980 \mathrm{~nm}$ and $1480 \mathrm{~nm}$ which combines the advantage of the high gain characteristics of $980 \mathrm{~nm}$ pumping with the broad bandwidth characteristics of $1480 \mathrm{~nm}$ pumping. By pumping at $980 \mathrm{~nm}$, they show that the peak value of gain is expected to be around $69 \mathrm{~dB}$ with a gain bandwidth of $\sim 10-15 \mathrm{~nm}$. For pumping at $1480 \mathrm{~nm}$, the models show that the peak value of gain decreased as compared to $980 \mathrm{~nm}$ pumping but the gain bandwidth increased to around 30$32 \mathrm{~nm}$. However, by cascading two EDFAs pumped separately at $980 \mathrm{~nm}$ and $1480 \mathrm{~nm}$, the overall peak value of gain increased to $\sim 180 \mathrm{~dB}$ and with a bandwidth of $\sim 35 \mathrm{~nm}$. Further, the cascaded gain can be made nearly uniform for the entire spectral range of $35 \mathrm{~nm}$ by using appropriate optical filters. (These subjects also proved to be an area of high audience interest, and there were a number of side discussions on possible future papers that could cover test result cross-comparisons and quality measures that might incorporate performance uniformity over wavelength and the use of special optical fibers as part of this process.) 
Alexey Lantsov (Institute of Automation and Control Processes of FEB RAS, and the Far Eastern State Technical University, Russia) presented a paper for a team that addresses processing the signals from a single-fiber intermode interferometer (SFMI) with a small number of excited modes by using an electronic correlation method. They showed that use of a diffusive scatterer allows the transformation of the radiation of the SFMI to a speckle field which can then be easily be processed. The value of the measurement error of SFMI elongation is shown to be $\pm 10 \mu \mathrm{m}$, while the working range of the SFMI deformation measurements is shown to be 0-160 $\mu \mathrm{m}$.

There were also a number of very excellent Poster Papers: Kevin L. Mahoney, et al. (Naval Oceanographic Office, along with authors from the Stennis Space Center, Rutgers University, Metron, Inc., and Wet Labs, Inc.) presented a poster on the effectiveness of sensors that use optical measurements for the laser detection and identification of subsurface mines as related to water clarity. The primary objective of the work presented was to use the optical data collected by unmanned underwater vehicle (Slocum Glider) surveys of an operational area to estimate the performance of an electro-optical identification (EOID) laser line scan (LLS) system during an international naval exercise off the coast of Hawaii. Measurements of optical backscattering and beam attenuation were made with a Wet Labs, Inc. Scattering Absorption Meter (SAM), mounted on a Rutgers University/Webb Research Slocum glider. The optical data universally indicated extremely clear water in the operational area, except very close to shore. The beam-c values from the SAM sensor were integrated to three attenuation lengths to provide an estimate of how well the LLS would perform in detecting and identifying mines in the operational areas. The optical data was transmitted via IRIDIUM and served as near-real-time input to the electro-optic detection simulator model for EOID performance prediction. Both methods of predicting LLS performance suggested a high probability of detection and identification. These predictions were validated by the actual performance of the LLS as the EOID system yielded imagery from which reliable mine identification could be made. Future plans discussed included repeating this work in more optically challenging water types to demonstrate the utility of pre-mission UUV surveys of operational areas as a tactical decision aid for planning EOID missions, leading up to airborne mine countermeasure (AMCM) missions.

Nasrin Ghanbari, et al. (University of Arizona) discussed the modeling/use of a folded holographic optical system as a building block for a compact imager. The design of the system allow for a thin optical configuration and a reduction in the size of the optical elements. In the folded design a transmission hologram in a slanted configuration folds the field of view of the lens and points it at a specific direction in the object space. The slanted geometry results in a compressed nonuniform field of view that is defined as a vignetting function and generated by a ray-tracing program. In the compact imaging system, multiple folded holographic imagers are used in an array to extend the field of view. Each imager unit was optimized to collect a segment of light from the object scene, 
and a combination of imager units defines the composite field of view of the compact imaging system. The folded holographic imager is capable of capturing a diffraction limited image with full-field angle of $30^{\circ}$ although it suffers from low SNR. It is expected that SNR can be improved by increasing the number of lens elements, reducing the compression factor, altering the structure of the folded imager, and modifying the shape of the system aperture. The need for image processing research to physically implement the system was also covered.

Elizabeth Matzinger (NASA Goddard Space Flight Center) presented a paper on the finite element stress and distortion analysis of the receiver telescope of the Lunar Orbiter Laser Altimeter (LOLA). LOLA is one of six instruments on the Lunar Reconnaissance Orbiter (LRO), scheduled to launch in 2008. LOLA's main objective is to produce a high-resolution global lunar topographic model to aid in safe landings and enhance surface mobility in future exploration missions. The receiver telescope captures the laser pulses transmitted through a diffractive optical element (DOE) and reflected off the lunar surface. The largest lens of the receiver telescope was modeled with solid elements and constrained in a manner consistent with the behavior of the mounting configuration. Twenty-one temperature load cases were mapped to the nodes based on thermal analysis, and loads were applied to simulate the preload applied from a ring flexure. The thermal environment of the baseline design produces large radial $\left(\sim 25^{\circ} \mathrm{C}\right)$ and axial $\left(\sim 10^{\circ} \mathrm{C}\right)$ gradients in the lens, and these large gradients were shown to create internal stresses that might lead to part failure, as well as significant bending and localized changes in refractive index that degrade optical performance. Bounding the hot transient case proved to be a challenge, as surprising stress results came from the 20 timesteps chosen in the second orbit. The first four timesteps chosen, those with the largest positive and negative radial and axial gradients, did include the case with maximum predicted distortion. However, the largest stress prediction occurred in a case with an axial gradient of $16.5^{\circ} \mathrm{C}$, less than the $21^{\circ} \mathrm{C}$ maximum, and a radial gradient of $-6.1^{\circ} \mathrm{C}$, less than half the most negative radial gradient of $15.9^{\circ} \mathrm{C}$. The high stresses and large distortions shown in the analysis precipitated a design change from BK7 glass to sapphire which was predicted to operate closer to the $20^{\circ} \mathrm{C}$ integration temperature, has no on-orbit gradients, and is a higher strength material.

Baotang Shan, et al. (Beijing Institute of Technology along with team members from Hong Kong Polytechnic University) were unfortunately not able to attend the conference due to factors stated to be beyond their control, but the team's paper on the design and application of a real-time demosaicing generic model based on a multiple channel CMOS image sampling system has been included in these proceedings. (The work is both new and detailed, and the authors have updated their paper to include inputs made in a conference-based refereed review of their work.). The real-time demosaicing generic model presented is concise, easy to implement, resource-saving, and robust. With this model the authors show that multiple displaying demosaicing subsystems can be realized in terms of a Bayer pattern color filter array. In this work, an LCD displaying 
subsystem is designed by FPGA, and the simulated waveform is presented. The monitoring results of a real scene are also captured and illustrated. To prove the validity of the LCD monitoring results, a real-world subsystem is discussed which uses a multiple channel CMOS image sampling system. Three images exposed synchronously in the background system and the actual pictures stored are shown, one of which is identical to the scene monitored by the LCD subsystem. Finally, to demonstrate the generality of the model, the test results of a CRT demosaicing displaying subsystem designed with the model are presented.

Last but not least, the full richness of application diversity and increasingly sophisticated operational requirements combine to make optical modeling and performance predictions an area where challenges abound. Clearly clever thinking can continue to return high intellectual rewards while significantly contributing to our collective ability to understand and improve the hardware of tomorrow.

Mark Kahan 
Downloaded From: https://www.spiedigitallibrary.org/conference-proceedings-of-spie on 26 Apr 2023

Terms of Use: https://www.spiedigitallibrary.org/terms-of-use 